\title{
ADVANCES IN APPLIED PROBABILITY VOLUME 44 (2012): INDEX General Applied Probability
}

Andreoli, A., Caravenna, F., Dai Pra, P. and Posta, G. Scaling and multiscaling in financial

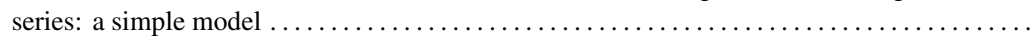

Balkema, G. And Nolde, N. Asymptotic dependence for light-tailed homothetic densities.......

BALL, F. AND SIRL, D. An SIR epidemic model on a population with random network and household



BAR-LEv, S. K. AND LETAC, G. Increasing hazard rate of mixtures for natural exponential families

BEGHIN, L. Fractional relaxation equations and Brownian crossing probabilities of a random boundary

Bhaskar, A. AND Song, Y. S. Closed-form asymptotic sampling distributions under the coalescent

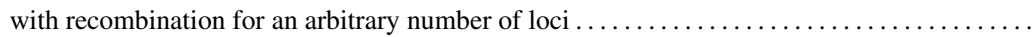

—, KAmm, J. A. AND Song, Y. S. Approximate sampling formulae for general finite-alleles models

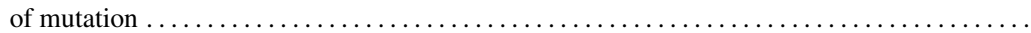

$391-407$

$408-428$

Brandejsky, A., De Saporta, B. and Dufour, F. Numerical methods for the exit time of a

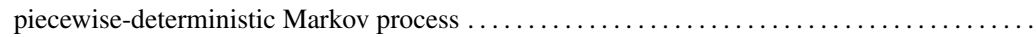

Caravenna, F. see Andreoli, A.

Ceci, C. AND Colaneri, K. Nonlinear filtering for jump diffusion observations . . . . . . . . . . .

Chan, H. P., Deng, S. AND LAI, T.-L. Rare-event simulation of heavy-tailed random walks by sequential importance sampling and resampling. ...

$196-225$

$678-701$

$1173-1196$

Chen, A., Li, J., Chen, Y. AND ZHou, D. Extinction probability of interacting branching collision

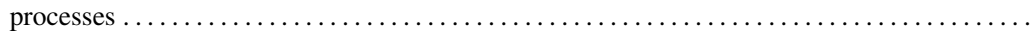

Chen, Y. see Chen, A.

CHI, Z. On exact sampling of nonnegative infinitely divisible random variables $\ldots \ldots \ldots \ldots \ldots \ldots$

Colaneri, K. see Ceci, C.

Czichowsky, C. AND Schweizer, M. Convex duality in mean-variance hedging under convex

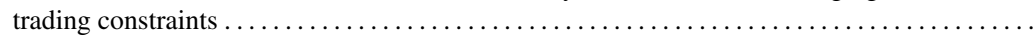

Dai Pra, P. see Andreoli, A.

Daly, F., Lefèvre, C. And Utev, S. Stein's method and stochastic orderings . .............

DaYAnIK, S. AND Egami, M. Optimal stopping problems for asset management $\ldots \ldots \ldots \ldots \ldots$.

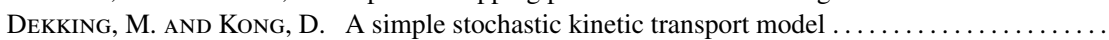

DENG, S. see CHAN, H. P.

Dereich, S., Mönch, C. And Mörters, P. Typical distances in ultrasmall random networks......

De Saporta, B. see Brandejsky, A.

Doumas, A. V. And Papanicolaou, V. G. The coupon collector's problem revisited: asymptotics of the variance.

Dufour, F., Horiguchi, M. And Piunovskiy, A. B. The expected total cost criterion for Markov decision processes under constraints: a convex analytic approach $\ldots \ldots \ldots \ldots \ldots \ldots \ldots \ldots$ see BRANDEJSKY, A.

Eder, I. ANd KlüPPElBerg, C. Pareto Lévy measures and multivariate regular variation .........

Egami, M. see DaYANIK, S.

FALK, M. AND Tichy, D. Asymptotic conditional distribution of exceedance counts ...........

Feuillet, M. AND Robert, P. On the transient behavior of Ehrenfest and Engset processes ......

Fontbona, J., Guérin, H. And Malrieu, F. Quantitative estimates for the long-time behavior of



Franz, U., Liebscher, V. ANd Zeiser, S. Piecewise-deterministic Markov processes as limits of Markov jump processes . 
Fuh, C.-D. AND PANG, T.-X. A self-normalized central limit theorem for Markov random walks . .

GÅSEMYR, J. Bounds for the availabilities of multistate monotone systems based on decomposition

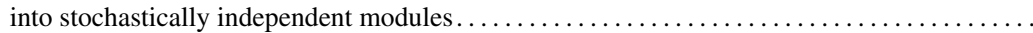

Genadot, A. And Thieullen, M. Averaging for a fully coupled piecewise-deterministic Markov



Ghosh, S. AND GHosh, S. A strong law for the rate of growth of long latency periods in a cloud

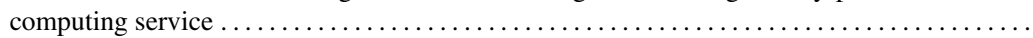

$995-1017$

GHosh, S. see GHosh, S.

GuÉrin, H. see Fontbona, J.

Hazra, R. S. AND Maulik, K. Tail behavior of randomly weighted sums ................

Horiguchi, M. see Dufour, F.

Jelenković, P. R. And Olvera-Cravioto, M. Implicit renewal theory and power tails on trees...

KAMM, J. A. see BHASKAR, A.

KeLLY, M. A hierarchical probability model of colon cancer $\ldots \ldots \ldots \ldots \ldots \ldots \ldots \ldots \ldots \ldots \ldots$

Kholfi, S. And Mahmoud, H. M. The class of tenable zero-balanced Pólya urn schemes:

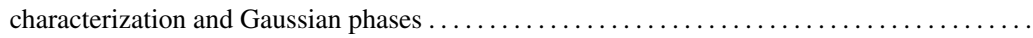

KlüPPELBERG, C. see EDER, I.

Kong, D. see DeKking, M.

Kuba, M. ANd PANholzer, A. Limiting distribution for a class of diminishing urn models .......

KULIK, R. AND SoULIER, P. Limit theorems for long-memory stochastic volatility models with infinite

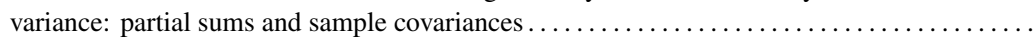

LAI, T.-L. see CHAN, H. P.

LefÈvre, C. see DALY, F.

Letac, G. see BAR-Lev, S. K.

LI, J. see CHEN, A.

Liebscher, V. see Franz, U.

LIN, K. AND REINERT, G. Joint vertex degrees in the inhomogeneous random graph model $g\left(n,\left\{p_{i j}\right\}\right)$

LIU, J. AND YANG, X. The convergence rate and asymptotic distribution of the bootstrap quantile variance estimator for importance sampling ...

Mahmoud, H. M. and Smythe, R. T. On the joint behavior of types of coupons in generalized

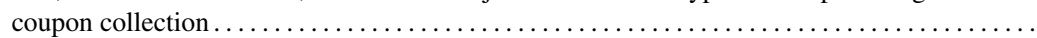

see KHOLFI, S.

Malrieu, F. see Fontbona, J.

MaUlik, K. see Hazra, R. S.

Mönch, C. see DEREICH, S.

Mörters, P. see Dereich, S.

Nadarajah, S. see Withers, C. S.

NEAL, P. Correction to Neal (2006, pp. 943-968) . . . . . . . . . . . . . . . . . . . . . .

Nolde, N. see Balkema, G.

Olvera-Cravioto, M. Asymptotics for weighted random sums

- see Jelenković, P. R.

PANG, T.-X. see FuH, C.-D.

Panholzer, A. see Kuba, M.

Papanicolaou, V. G. see Doumas, A. V.

Piunovskiy, A. B. see Dufour, F.

Posta, G. see Andreoli, A.

Reinert, G. see Lin, K.

Robert, P. see Feuillet, M.

Schweizer, M. see Czichowsky, C.

SirL, D. see BALL, F.

Smythe, R. T. see Mahmoud, H. M.

Song, Y. S. see BHASKar, A.

SOUlier, P. see Kulik, R.

Thieullen, M. see Genadot, A.

Tichy, D. see FALK, M.

Utev, S. see DALY, F. 
WANG, R. see WEI, J.

WEI, J., WANG, R. AND YANG, H. On the optimal dividend strategy in a regime-switching diffusion

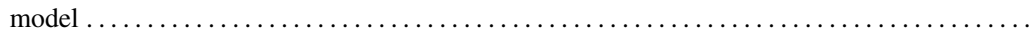

Withers, C. S. AND NADARAJAh, S. The distribution of Foschini's lower bound for channel capacity $886-906$

YANG, H. see WEI, J.

YANG, X. see LIU, J.

ZeISER, S. see Franz, U.

Zhou, D. see Chen, A. 